Article

\title{
Rotational Efficiency of Photo-Driven Archimedes Screws for Micropumps
}

\author{
Chih-Lang Lin ${ }^{1, *}$, Yu-Sheng Lin ${ }^{1}$ and Patrice L. Baldeck ${ }^{2}$ \\ 1 Biomedical Engineering and Materials Science, Central Taiwan University of Science and \\ Technology, 406 Taichung, Taiwan; E-Mail: m09905009@ms3.ctust.edu.tw \\ 2 University Grenoble 1, CNRS, LIPhy UMR 5588, 38402 Grenoble, France; \\ E-Mail: patrice.baldeck@ujf-grenoble.fr \\ * Author to whom correspondence should be addressed; E-Mail: cllin101943@ctust.edu.tw; \\ Tel.: +886-4-2239-1647 (ext. 6803).
}

Academic Editor: Joost Lötters

Received: 13 April 2015 / Accepted: 4 June 2015 / Published: 9 June 2015

\begin{abstract}
In this study, we characterized the rotational efficiency of the photo-driven Archimedes screw. The micron-sized Archimedes screws were fabricated using the two-photon polymerization technique. Free-floating screws trapped by optical tweezers align in the laser irradiation direction and rotate spontaneously. The influences of the screw pitch and the number of screw blades have been investigated in our previous studies. In this paper, the blade thickness and the central rod of the screw were further investigated. The experimental results indicate that the blade thickness contributes to rotational stability, but not to rotational speed, and that the central rod stabilizes the rotating screw but is not conducive to rotational speed. Finally, the effect of the numerical aperture (NA) of the optical tweezers was investigated through a demonstration. The NA is inversely proportional to the rotational speed.
\end{abstract}

eywords: Archimedes screw; optical tweezers; two-photon polymerization; optical torque; micropump

\section{Introduction}

Recently, two-photon polymerization (TPP) microfabrication has become popular for the manufacturing of three-dimensional (3D) microstructures. Essentially, it uses 3D laser direct writing 
technology to model arbitrary 3D complex microstructures [1-3] by scanning a laser-focusing point along predetermined trajectories. The TPP technique can accurately fabricate complex microstructures that can be manipulated by optical tweezers generated by highly focused laser beams without mechanical contact [4]. A series of photo-driven micromachines have been proposed in recent years using this technique [5-9]. Objects with anisotropic shapes can rotate around the laser axis due to unsymmetrical forces generated at their surface [10]. Such photo-driven rotation can be induced either from the net optical torque resulting from the complex shape [11,12] or by driving a movable part with an optical tweezers trap [13]. Recent works have made complex devices employed in microfluidic applications [14-18].

The Archimedes screw has been used in mechanical applications for 300 years. It efficiently transports fluid in a hollow, unclogged channel. Previously, we demonstrated that photo-driven Archimedes screws [10,19] can be rotated by optical tweezers and that the screw rotates spontaneously around its long axis when trapped at the laser focal point. This laser-induced rotation is due to the optical torque that is transferred by the laser scattering on the screw. The rotational speed is linearly proportional to the optical power. The influences of the screw pitch and the number of screw blades on the rotational speed of photo-driven Archimedes micro-screws have been studied and it has been found that screws with shorter pitches rotate slower. The rotational speed of a two-blade screw is twice the rotational speed of a one-blade screw. However, the three-blade screw rotates slower than 2-blade screw to the limited fabrication resolution.

In this study, we extended previous works to complete the influence of screw geometry, including blade thickness and the central rod of the screw. Furthermore, the effect of the numerical aperture (NA) of the optical tweezers was demonstrated.

\section{Fabrication of Archimedes Micro-Screw}

The Archimedes micro-screws were fabricated using a commercial TPP 3D-microfabrication machine (Teem Photonics Inc., Meylan, France) with a passively Q-switched Nd-YAG microchip laser (532 nm wavelength). The laser beam was expanded by a telescope coupled to an inverted microscope (Olympus IX51, Olympus Inc., Tokyo, Japan) and focused through a set of microscope objective lens $(\mathrm{NA}=1.3)$. Figure 1 shows the TPP fabrication system. First, a commercial resin (Photomer 3015 from Henkel, Düsseldorf, Germany) with 1\% photo-initiator was used for two-photon polymerization, which occurred in the resin at the focal point at about $0.15 \mathrm{~mW}$ laser power and after $1 \mathrm{~ms}$ of exposure time. A three-axis piezoelectric stage (Nanocube, Physik Ins., Karlsruhe, Germany) was used to translate the sample through the laser focus in order to produce the required structures. Finally, an ethanol (75\%) solution was added to dissolve the unexposed resin and to free the polymerized micro-objects in the chamber (10 mm diameter) that are made with two cover slips spaced by a double-faced tape.

In this paper, two types of Archimedes screws (with/without central rod) were proposed for investigating the effect of screw geometry. Figure 2 describes the geometry of an Archimedes screw. The height of the screw is $7.2 \mu \mathrm{m}$. The screw-turn number is 1.5 . The screw diameter is $3.2 \mu \mathrm{m}$. Figure 3 shows the scanning electron microscope (SEM) figures of polymerized products. 


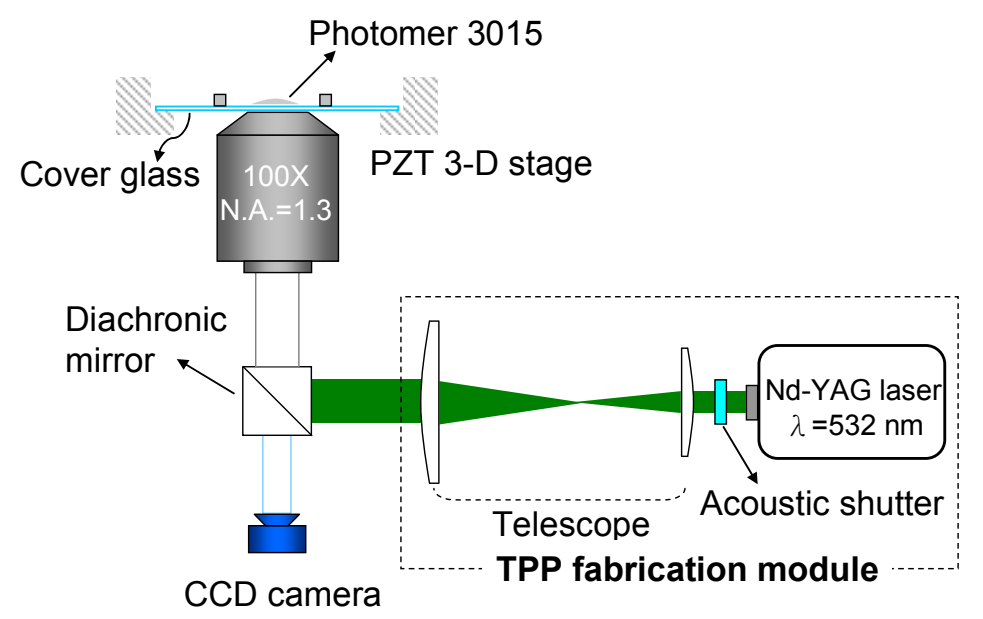

Figure 1. The two-photon polymerization (TPP) fabrication system.

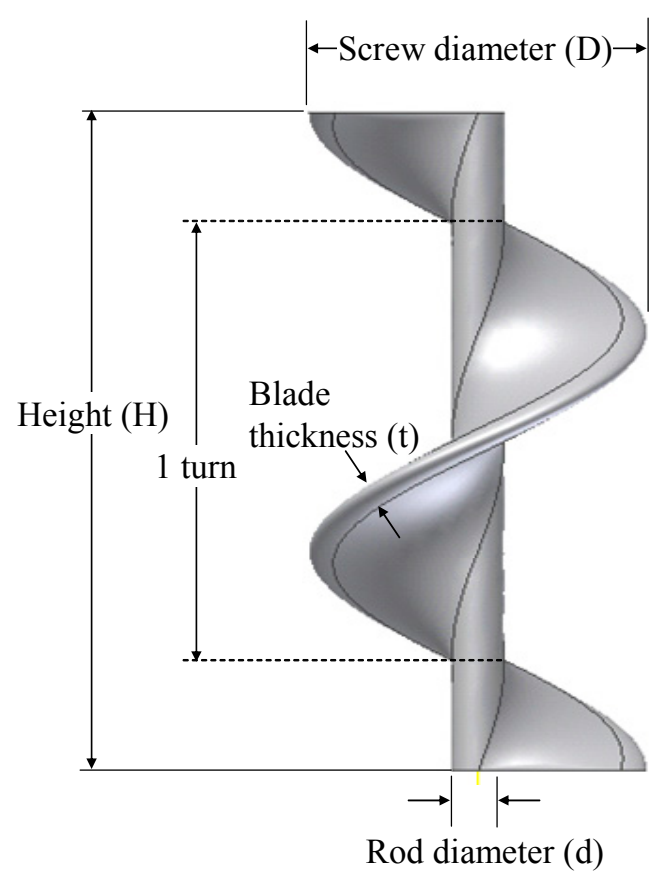

Figure 2. Geometric description of an Archimedes screw.

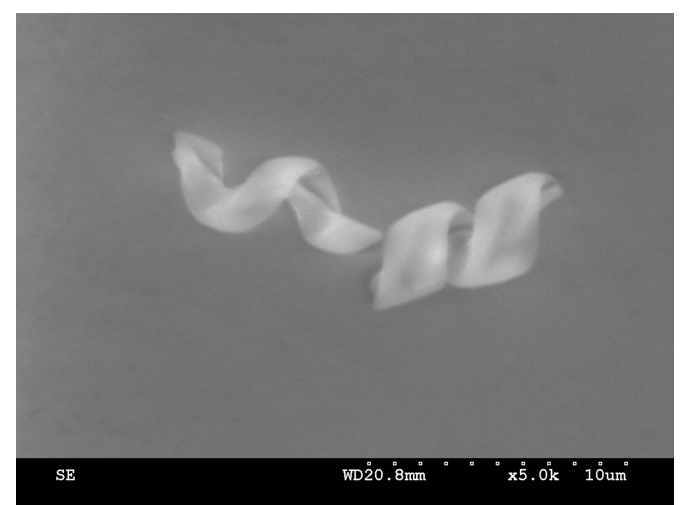

(a)

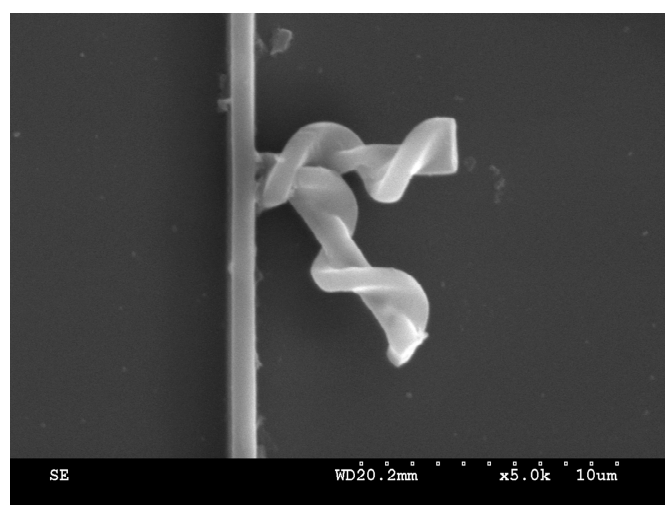

(b)

Figure 3. Scanning electron microscope (SEM) figures of polymerized products (Archimedes micro-screws): (a) screws without a central rod; (b) screws with a central rod. 


\section{Demonstration of Photo-Driven Archimedes Micro-Screws}

\subsection{Effect of Blade Thickness}

The typical behavior of an Archimedes micro-screw trapped by an optical tweezers was shown in our previous paper [19]. Figure 4 shows the optical tweezers system. The rotational speed was measured by using a photodiode to detect the modulated light transmitted through the micro-screw. An oscilloscope was used to visualize the photodiode signal. Each of the rotational speeds was determined after the rotation stabilized. In this experiment, measurements were performed with micro-screws of different blade thicknesses $(t)$, namely, $0.5 \mu \mathrm{m}, 1.0 \mu \mathrm{m}, 1.3 \mu \mathrm{m}$, and $1.6 \mu \mathrm{m}$ (Figure 5).

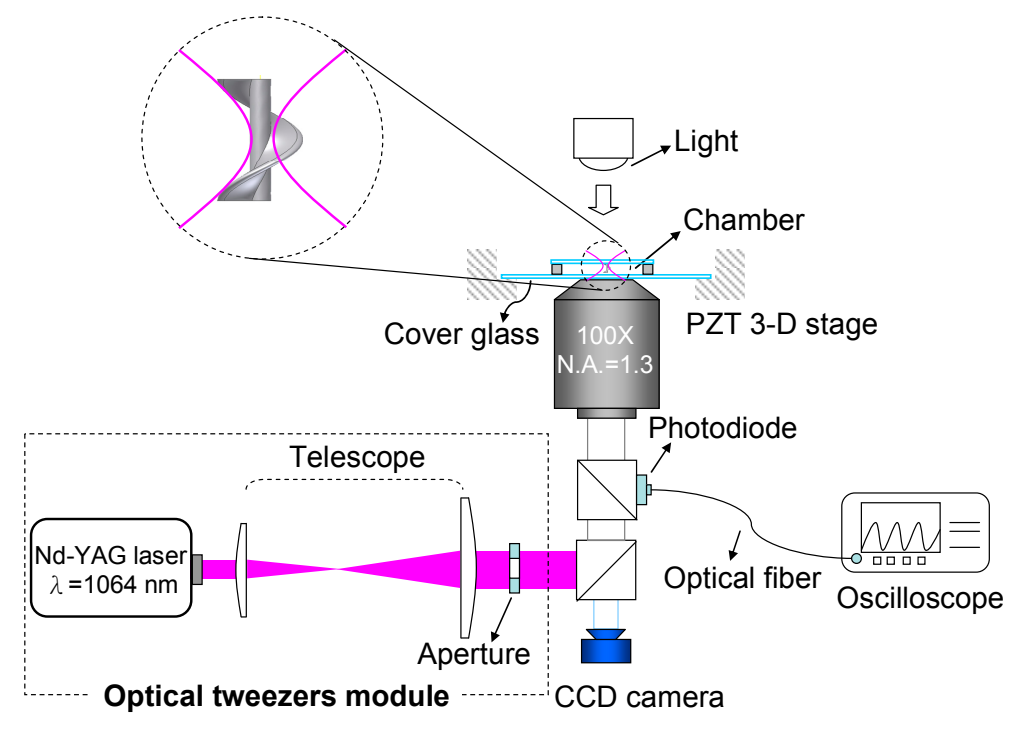

Figure 4. Diagram of the optical tweezers system.

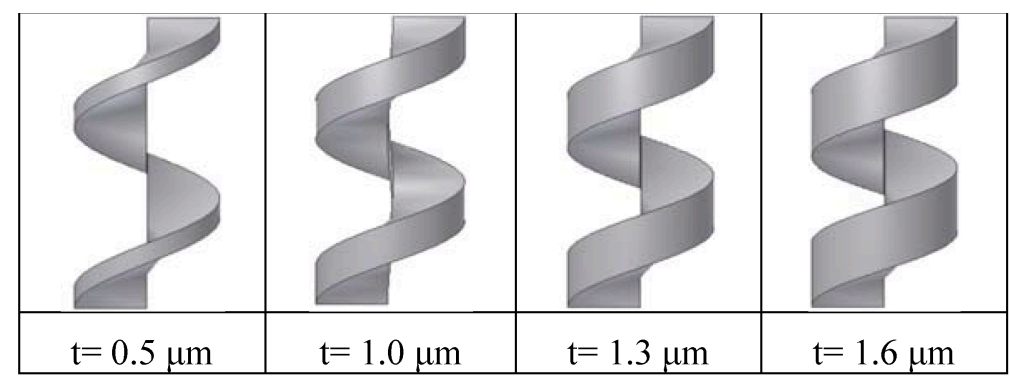

Figure 5. Computer Aided Design (CAD) of Archimedes screws without a central rod.

Figure 6 shows the relationship between rotational speed and laser power after a power threshold of $5 \mathrm{~mW}$. The experiment results show that the thin-blade screw $(t=0.5 \mu \mathrm{m})$ was trapped without rotation. The screw with $1.0 \mu \mathrm{m}$ thick blade rotated vacillatingly without aligning its axis in the laser direction; thus, the rotational speed was not linearly proportional to laser power. Only the screws with blade thicknesses of $1.3 \mu \mathrm{m}$ and $1.6 \mu \mathrm{m}$ rotated stably and aligned in the laser direction. In addition, it appeared that once the screws could rotate stably, the rotational speeds became constant. In other words, in this study, blade thickness only contributed to rotational stability and not to rotational speed. This is probably due to the fact that elongated objects in optical traps are known to align along the laser beam axis [20,21]. Figure 7 shows a schematic explanation of the influence of blade thickness. 


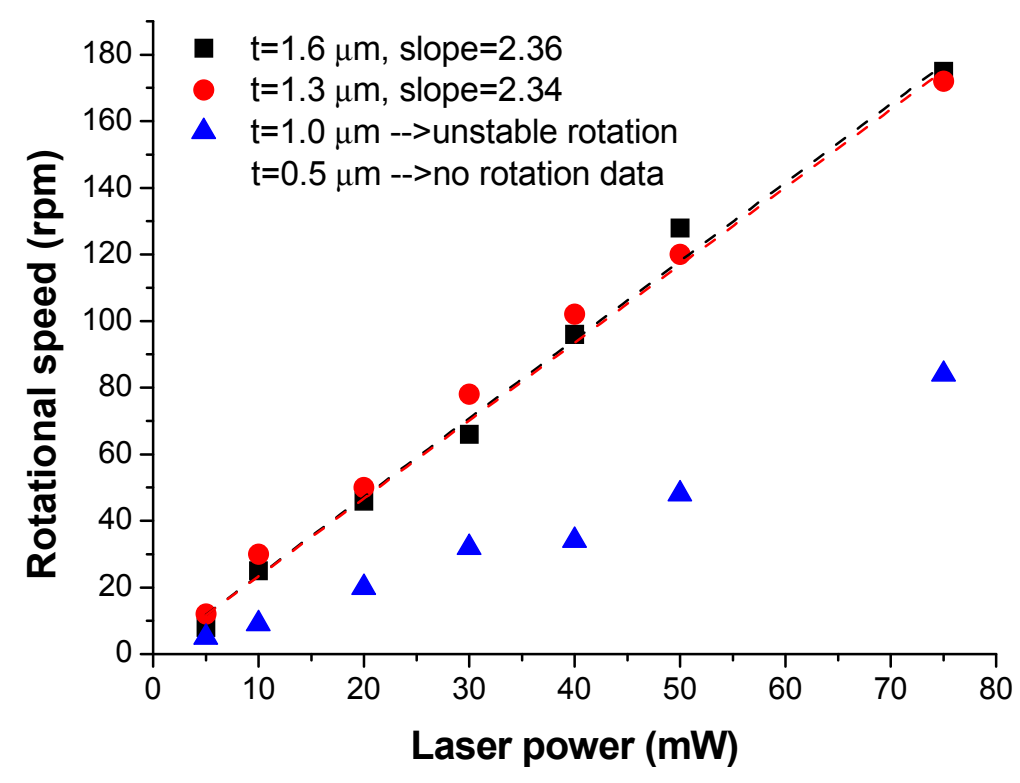

Figure 6. The evolution of rotational speeds under increasing laser power for micro-crews with different blade thicknesses $(t=0.5 \mu \mathrm{m}, 1.0 \mu \mathrm{m}, 1.3 \mu \mathrm{m}$, and $1.6 \mu \mathrm{m})$.

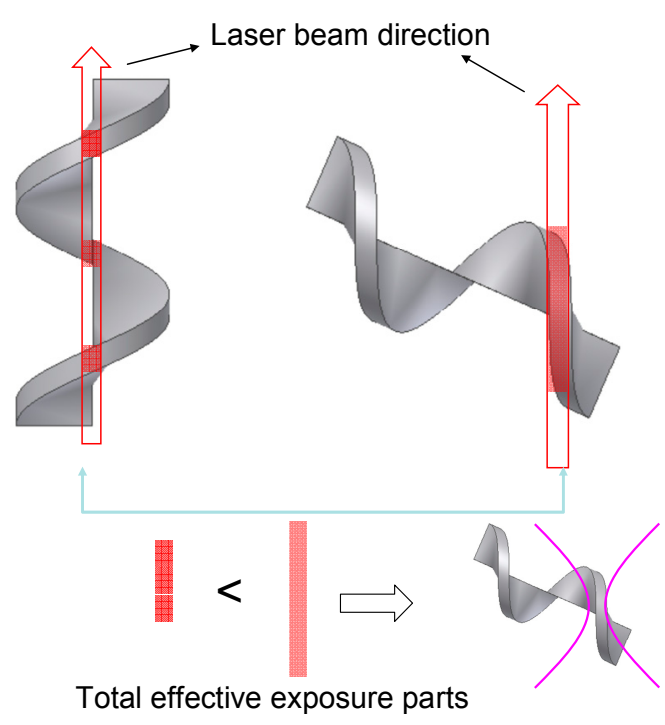

(a)

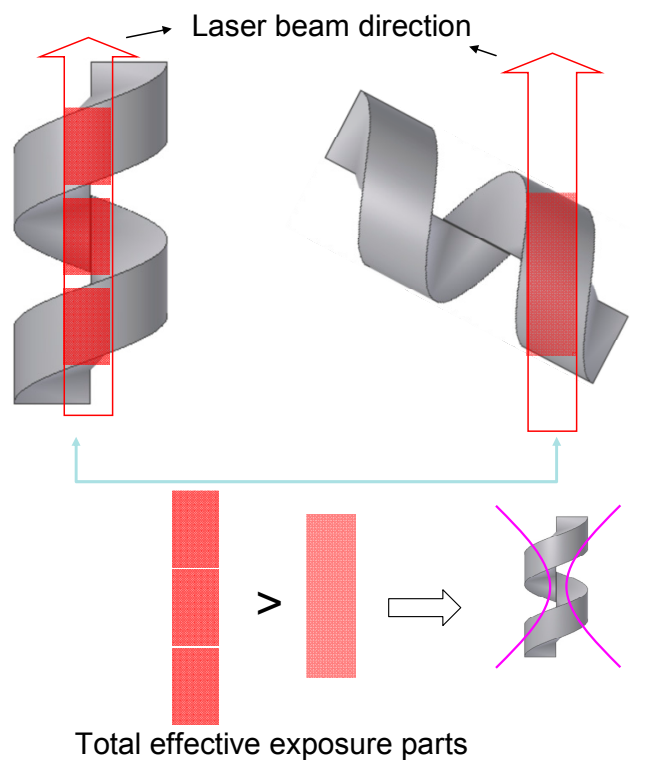

(b)

Figure 7. Schematic explanation of the influence of blade thickness: (a) thin-blade screw; (b) thick-blade screw.

\subsection{Effect of the Central Rod}

To stabilize the rotation of the thin-blade screw, a rod was added at the central axis of the screw (Figure 8). In this experiment, measurements were performed for micro-screws with different central rod diameters, namely, 0.5, 0.7, 0.9, and $1.1 \mu \mathrm{m}$. Figure 9 shows that the rotational speeds for each of the different rod diameters are linearly proportional to the laser power. As expected, even if the blade was very thin $(t=0.5 \mu \mathrm{m})$, screws with a central rod could rotate stably. In other words, a central rod allows an elongated object in an optical trap to align along the laser axis. However, screws with larger rod diameters rotate at slower rotational speeds. As the rod size increases, the proportional size of the 
blades decreases, reducing the effective exposure area for driving the screw. Figure 10 illustrates the effective exposure area to be exerted by the optical tweezers. L was defined as screw length. Thus, the effective exposure area can be expressed as $(D / 2-d / 2) \times$ L. Figure 11 shows the rotational efficiency versus the effective exposure area. Rotational efficiency is defined as the ratio of the micro-screw rotational speeds $(\mathrm{rpm})$ to the laser power $(\mathrm{mW})$. The high coefficient of determination $\left(R^{2}=0.996\right)$ indicates that the experimental results coordinate well with our inferences.

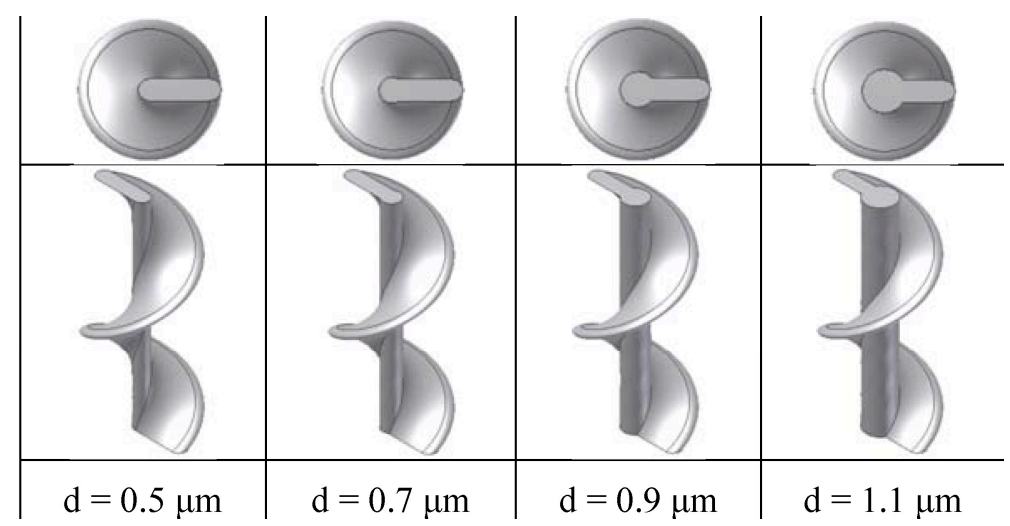

Figure 8. AutoCAD designs of Archimedes screws with central rod.

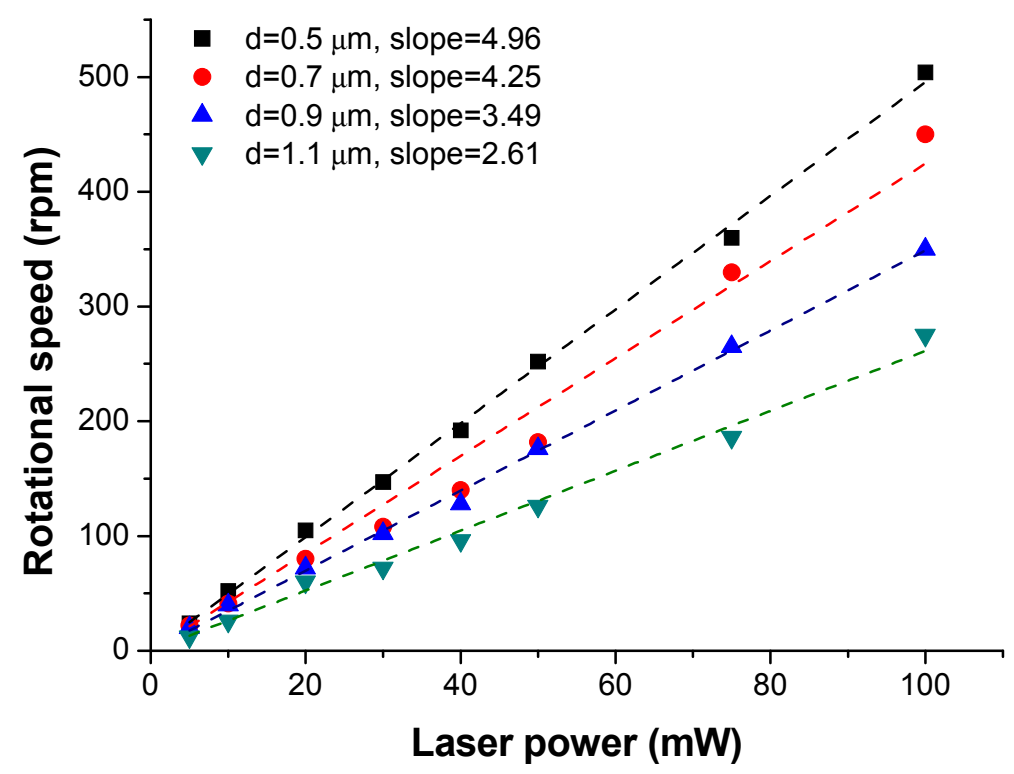

Figure 9. The evolution of rotational speeds under increasing laser power for micro-crews with different rod diameters $(d=0.5 \mu \mathrm{m}, 0.7 \mu \mathrm{m}, 0.9 \mu \mathrm{m}$, and $1.1 \mu \mathrm{m})$.

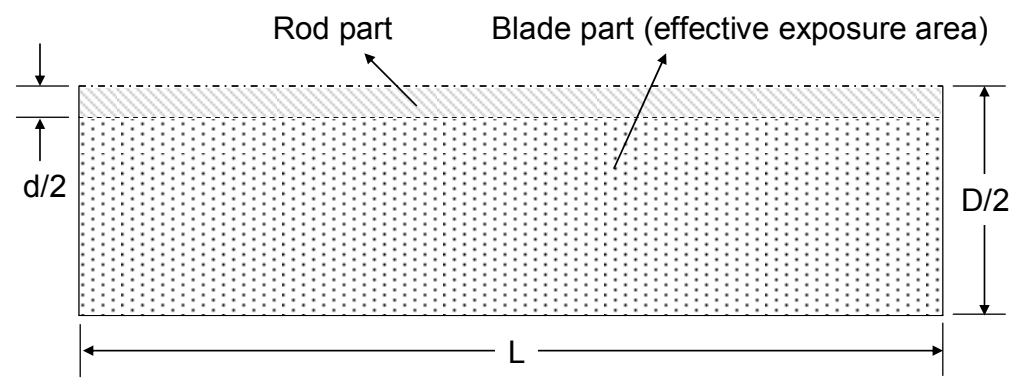

Figure 10. Diagram of effective exposure area. 


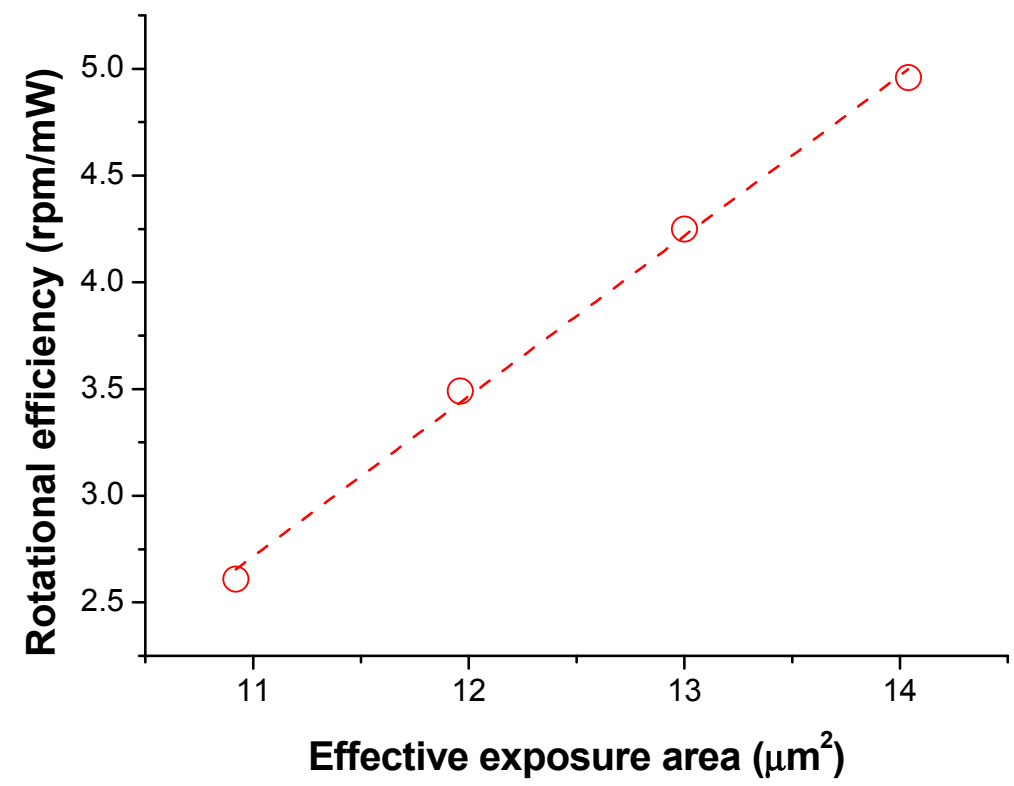

Figure 11. Rotational efficiency versus effective exposure area.

\subsection{Effect of the Numerical Aperture}

Essentially, the optical trap includes a gradient force and a scattering force. It is known that the gradient force dominates the lateral trapping and the scattering force dominates the axial pushing. In addition, a small NA forms a large scattering force which results in more axial pushing on the screw blade in order to generate a high rotational speed. Figure 12 illustrates the relevance of the NA, axial pushing, and rotational speed. In this experiment, a mechanical aperture (Figure 4) was used to adjust the NA. Archimedes screws were trapped using optical tweezers with a series of NAs $(1.2,1.23,1.27$, and 1.3). The results are shown in Figure 13. Figure 14 shows the rotational efficiency versus 1/NA. The high coefficient of determination $\left(R^{2}=0.987\right)$ indicates that the experimental results coordinate well with our inferences.

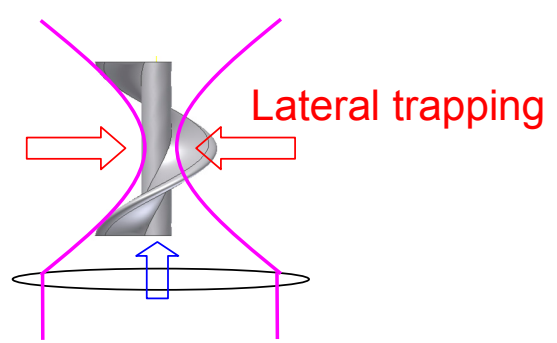

Axial pushing

(a)

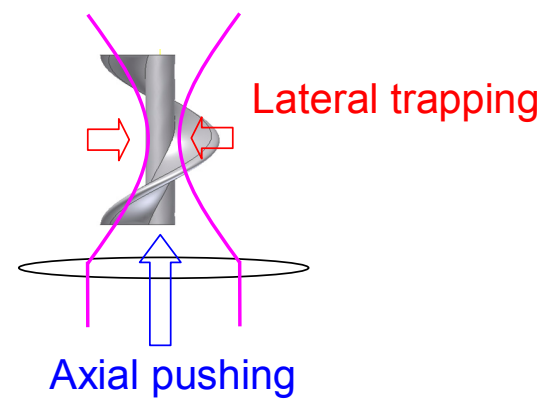

(b)

Figure 12. Two cases of optical trappings: (a) large numerical aperture (NA); (b) small NA. 


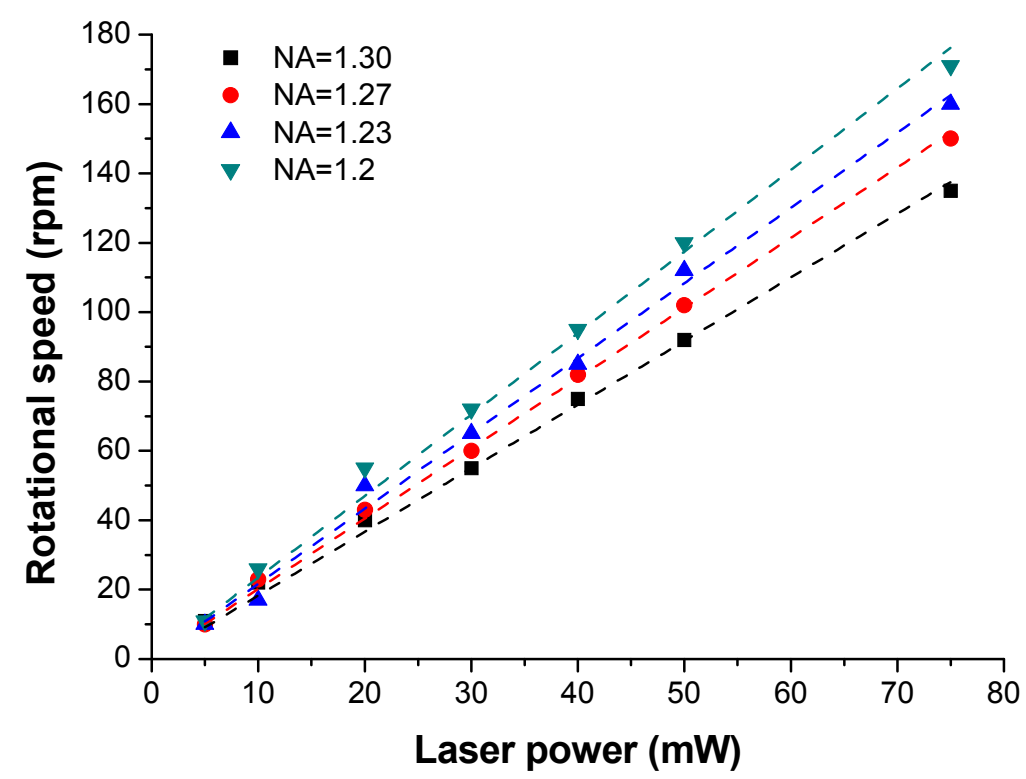

Figure 13. The evolution of rotational speeds under increasing laser power for micro-crews with different NAs (NA $=1.3 \mu \mathrm{m}, 1.27 \mu \mathrm{m}, 1.23 \mu \mathrm{m}$, and $1.2 \mu \mathrm{m}$ ).

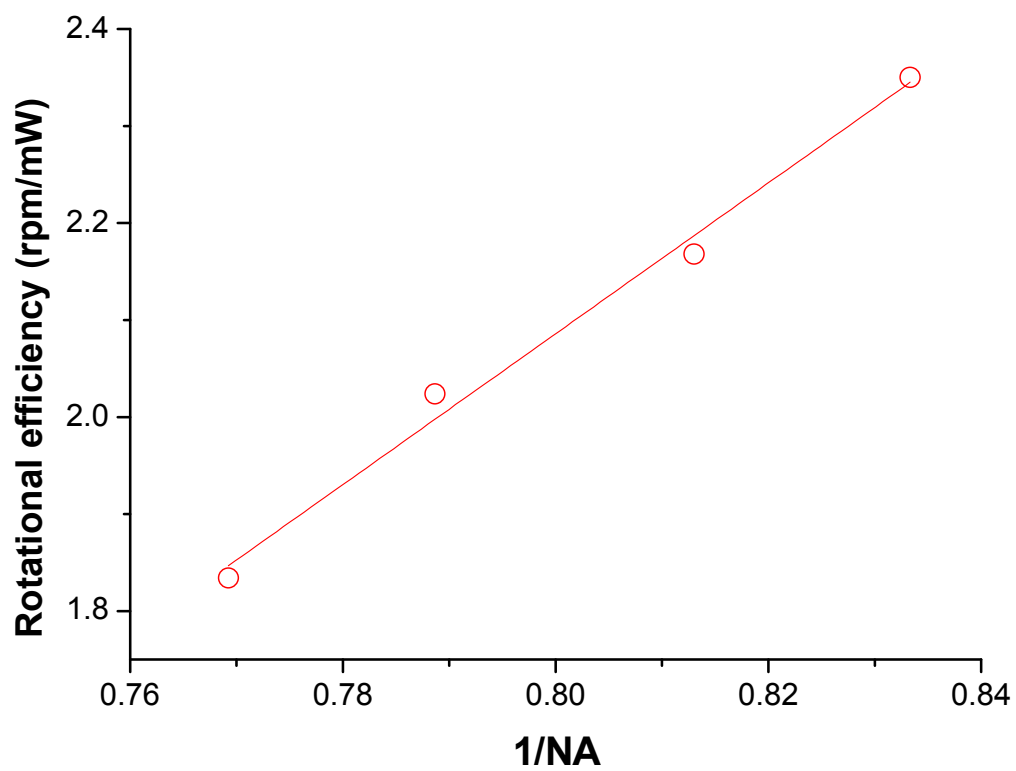

Figure 14. Rotational efficiency versus 1/NA.

\section{Conclusions}

A series of studies on photo-driven Archimedes screws have been made in our works. The rotational efficiency of a photon-driven Archimedes screw is mainly determined by the screw geometry and the numerical aperture (NA) of the optical tweezers. The influence of screw geometry includes the screw number, the blade number, the blade thickness, and the central rod. In conclusion, a small pitch and thin blades contribute to the rotational speed. The central rod stabilizes the screw rotation. However, larger rod diameters correlate to slower rotational speeds. Finally, a high NA of the optical tweezers is conductive to stably trapping a micro-screw. In contrast, a small NA contributes to the rotational speed. Table 1 summarizes the influences of screw geometry and the NA of the optical tweezers. 
Table 1. Qualitative summary of the influences of screw geometry and the NA.

\begin{tabular}{cccccc}
\hline Parameters & Screw Number & Blade Number & Blade Thickness & Central Rod & NA \\
\hline Rotational speed & $\downarrow$ & $\uparrow$ & - & $\downarrow$ & $\downarrow$ \\
Stabilization & - & - & $\uparrow$ & $\uparrow$ & $\uparrow$ \\
\hline
\end{tabular}

Notes: $\downarrow$ : negative, $\uparrow:$ positive, - : no effect.

\section{Acknowledgments}

This research was supported by the Ministry of Science and Technology (MOST 103-2221-E-166-008 \& MOST 103-2633-E-166-001).

\section{Author Contributions}

Chih-Lang Lin conceived and designed the experiments; Yu-Sheng Lin performed the experiments; Chih-Lang Lin and Patrice L. Baldeck analyzed the data; Chih-Lang Lin wrote the paper.

\section{Conflicts of Interest}

The authors declare no conflict of interest.

\section{References}

1. Maruo, S.; Nakamura, O.; Kawata, S. Three-dimensional microfabrication with two-photon-absorbed photopolymerization. Opt. Lett. 1997, 22, 132-134.

2. Joshi, M.P.; Pudavar, H.E.; Swiatkiewicz, J.; Prasad, P.N.; Reinhardt, B.A. High-density three-dimensional optical data storage in a stacked compact disk format with two-photon writing and single photon readout. Appl. Phys. Lett. 1999, 74, 1338-1340.

3. Sun, H.B. Matsuo, S.; Misawa, H. Three-dimensional photonic crystal structures achieved with two-photon-absorption photopolymerization of resin. Appl. Phys. Lett. 1999, 74, 786-788.

4. Ashkin, A.; Dziedzic, J.; Bjorkholm, J.; Chu, S. Observation of a single-beam gradient force optical trap for dielectric particles. Opt. Lett. 1986, 11, 288-290.

5. Moffitt, J.R.; Chemla, Y.R.; Smith, S.B.; Bustamante, C. Recent advances in optical tweezers. Annu. Rev. Biochem. 2008, 77, 205-228.

6. Wang, J.; Xia, H. Xu, B.B.; Niu, L.G.; Wu, D.; Chen, Q.D.; Sun, H.B. Remote manipulation of micronanomachines containing magnetic nanoparticles. Opt. Lett. 2009, 34, 581-583.

7. Lin, L.; Fei, Z.; Lu, H.; Li, Z.Y. Optical forces on arbitrary shaped particles in optical tweezers. J. Appl. Phys. 2010, 108, 073110.

8. Lin, C.L.; Lee, Y.H.; Lin, C.T.; Liu, Y.-J.; Hwang, J.L.; Chung, T.T.; Baldeck, P.L. Multiplying optical tweezers force using a micro-lever. Opt. Express 2011, 19, 20604-20609.

9. Lin, C.L.; Li, Y.H.; Lin, C.T.; Chiang, C.C.; Liu, Y.J.; Chung, T.T.; Baldeck, P.L. Preliminary study of lever-based optically driven micro-actuator. In Proceedings of the 3rd International Conference on Smart Materials and Nanotechnology in Engineering, Shenzhen, China, 5-8 December 2011. 
10. Baldeck, P.L.; Lim, C.L.; Lin, Y.S.; Lin, C.T.; Chung, T.T.; Bouriau, M.; Vitrant, G. Optically driven Archimedes micro-screws for micropump applications: Multiple blade design. SPIE Proc. 2011, 8097, doi:10.1117/12.893679.

11. Galadja, P.; Ormos, P. Complex micromachines produced and driven by light. Appl. Phys. Lett. 2001, 78, 249-251.

12. Galadja, P.; Ormos, P. Rotors produced and driven in laser tweezers with reversed direction of rotation. Appl. Phys. Lett. 2002, 80, 4653-4655.

13. Ikuta, K.; Sasaki, Y.; Maegawa, L.; Maruo, S. Biochemical IC chip for pretreatment in biochemical experiments. In Proceedings of the IEEE 6th Annual International Conference on Micro Electro Mechanical Systems, Kyoto, Japan, 19-23 January 2003; pp. 343-346.

14. Maruo, S.; Inoue, H. Optically driven micropump produced by three-dimensional two-photon microfabrication. Appl. Phys. Lett. 2006, 89, 144101.

15. Maruo, S.; Inoue, H. Optically driven viscous micropump using a rotating microdisk. Appl. Phys. Lett. 2007, 91, 084101.

16. Maruo, S.; Takaura, A.; Saito, Y. Optically driven micropump with a twin spiral microrotor. Opt. Express 2009, 17, 18525-18532.

17. Liu, Y.J.; Lee, Y.H.; Lin, Y.S.; Tsou, C.; Baldeck, P.L.; Lin, C.L. Optically Driven Mobile Integrated Micro-tools for Lab-on-a-chips. Actuators 2013, 2, 19-26.

18. Lee, Y.H.; Liu, Y.J.; Tzou, C.F.; Bouriau, M.; Baldeck, P.L.; Lin, C.L. Optically driven gear-based mechanical micro-transducer for lab-on-a-chip. J. Neurosci. Neuroeng. 2013, 2, 58-60.

19. Lin, C.L.; Vitrant, G.; Bouriau, M.; Casalegno, R.; Baldeck, P.L. Optically driven Archimedes microscrews for micropump applications. Opt. Express 2011, 19, 8267-8276.

20. Nieminen, T.A.; Rubinsztein-Dunlop, H.; Heckenberg, N.R. Angular momentum generation by scattering: Alignment and rotation of microobjects. In Proceedings of the 6th Conference on Electromagnetic and Light Scattering by Nonspherical Particles: Theory, Measurements, and Applications, Gainesville, FL, USA, 4-8 March 2002; pp. 239-242.

21. Lin, C.L.; Wang, I.; Pierre, M.; Colombier, I.; Andraud, C.; Baldeck, P.L. Rotational properties of micro-slabs driven by linearly polarized light. J. Nonlinear Opt. Phys. Mater. 2005, 14, 375-382.

(C) 2015 by the authors; licensee MDPI, Basel, Switzerland. This article is an open access article distributed under the terms and conditions of the Creative Commons Attribution license (http://creativecommons.org/licenses/by/4.0/). 